\title{
Pulmonary restrictive effect of bracing in mild idiopathic scoliosis
}

\author{
J D KENNEDY, C F ROBERTSON, A OLINSKY, D R V DICKENS, P D PHELAN \\ From the Professorial Department of Thoracic Medicine and Department of Orthopaedics, Royal Children's \\ Hospital, and Department of Paediatrics, University of Melbourne, Melbourne, Australia
}

\begin{abstract}
The use of bracing in the treatment of mild idiopathic scoliosis is controversial. A study of 33 adolescents showed that bracing significantly decreased lung volumes. Functional residual capacity was reduced by a mean of $26 \%, 18 \%$ of children showing a reduction of greater than $40 \%$. The mean reduction in total lung capacity was $16 \%$ and in forced vital capacity $18 \%$. This restriction of lung function by bracing might have a deleterious effect on lung growth or might impose an additional risk factor in the presence of other disorders, such as asthma and diaphragmatic weakness. The use of bracing in individuals with mild scoliosis should be judiciously reassessed.
\end{abstract}

Scoliosis is one of man's earliest known physical deformities. It has been depicted in cave drawings, noted in the mummified remains of the pharaohs, and aroused fear in Victor Hugo's tale of Quasimodo, the Hunchback of Notre Dame.

Despite this long history, its treatment, particularly bracing, remains controversial. The use of bracing in the management of scoliosis began during the 1940s with the introduction of the Milwaukee brace, designed to stabilise the spine postoperatively. ${ }^{1}$ During the 1950s and 1960s bracing began to be used in the non-operative management of scoliosis. During the 1970s the Boston brace was introduced. ${ }^{2}$ There is continuing debate in orthopaedic publications about the indications and effectiveness of bracing programmes. ${ }^{3,4}$

Although braces have been used extensively in the past 30 years, their effect on underlying lung function and chest wall mechanics is largely unknown. We report the effect of bracing on lung volumes in 33 adolescents with idiopathic scoliosis.

\section{Patients and methods}

We studied 33 adolescents, six male and 27 female, with idiopathic thoracic scoliosis attending an orthopaedic clinic and being treated by bracing. Eight used a Milwaukee brace and 25 a Boston brace. Their mean

\footnotetext{
Address for reprint requests: Dr C F Robertson, Professorial Department of Thoracic Medicine, Royal Children's Hospital, Parkville 3052, Victoria, Australia.

age was $13 \cdot 3$ years and the mean thoracic Cobb angle $29^{\circ}$. In five children the thoracic curve had improved with bracing and at the time of testing was less than 20 degrees. The mean age of onset was 11.7 years (range 6-16 years) and the mean duration of bracing 1.4 years (range 1 month-5 years). None of the patients had cardiopulmonary symptoms at the time of testing. Functional residual capacity (FRC) and total lung capacity (TLC) were measured in the sitting position by means of body plethysmography (Jaeger Bodyscreen 2) both with and without the brace. The order of testing - that is, whether the brace was on or off for the first measurements - was randomly selected to allow for a learning effect. All spirometric volumes were corrected to BTPS. The non-deformed height was taken as equal to arm span. In our laboratory the armspan:height ratio estimated from 2368 measurements of 8-18 year old subjects is close to unity $(0.999$ (SD 0.023) for girls and 1.006 (0.022) for boys).

The Milwaukee brace reduces lumbar lordosis with a polypropylene pelvic girdle and attempts to correct the thoracic curve with a metal vertical suprastructure on which are mounted pressure pads. The Boston bracing system also uses a polypropylene girdle, which, unlike the former, extends to enclose the lower thorax, again attempting to correct the spinal curve by means of pressure pads mounted on the inner surface, acting on the apices of the curve.

Six children with a brace induced reduction in FRC of over $30 \%$ were further studied to assess the effect of bracing on oxygen saturation during sleep. Oxygen saturation was monitored overnight with a Nelcor pulse oximeter; studies were performed on two nights, one with and the other without the brace. 
We used analysis of variance, repeated measures design, ${ }^{5}$ to assess the effect of gender and brace type on reduction in lung volumes; $p$ values of less than 0.05 were regarded as significant.

The study was approved by the hospital ethics committee and informed consent was obtained from subjects and parents.

\section{Results}

As a group, the subjects had normal lung volumes when unbraced (table 1). Although some patients had a mild restrictive defect, this did not correlate with age of onset of scoliosis or duration of bracing.

With the brace on, there were significant reductions in all the subdivisions of total lung capacity, especially

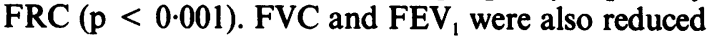
$(p<0.001)$ (table 2). The 95th centiles for coefficient of variation for lung volumes in normal subjects from our laboratory are included in the table for comparison. The mean reduction in FRC was $26 \%$. Six children (18\%), however, had a reduction of over $40 \%$ and 13 children $(39 \%)$ had a reduction of at least $30 \%$. No difference was found between the restrictive effect of the brace on boys and on girls. There was also no difference between the restrictive effects on lung volumes of the Boston and the Milwaukee brace.

Table 1 Details of subjects and their lung function while unbraced

\begin{tabular}{lll}
\hline & Male & Female \\
\hline Number & 6 & 27 \\
Age $(\mathrm{y})^{*}$ & $12 \cdot 4(11 \cdot 0-15 \cdot 0)$ & $13 \cdot 8(10 \cdot 0-17 \cdot 0)$ \\
Arm span $(\mathrm{cm})^{*}$ & $154(132-184)$ & $164(140-178)$ \\
Thoracic Cobb angle $\left(^{\circ}\right)^{*}$ & $32(20-40)$ & $28(10-44)$ \\
${\text { Age at onset }(\mathrm{y})^{*}}^{\text {Duration of bracing }(\mathrm{y})^{*}}$ & $8(6-13)$ & $13(7-16)$ \\
Lung function $(\%$ predicted) $\dagger$ & $3 \cdot 3(0 \cdot 7-5)$ & $0 \cdot 9(0 \cdot 1-3 \cdot 8)$ \\
VC & $87(10)$ & $88(13)$ \\
FEV & $82(10)$ & $86(13)$ \\
FRC & $97(18)$ & $103(17)$ \\
TLC & $93(9)$ & $99(12)$ \\
\hline
\end{tabular}

*Median and range.

†Mean and standard deviation.

VC-vital capacity; FEV - forced expiratory volume in one second; FRC - functional residual capacity; TLC - total lung capacity.

Table 2 Effects of bracing on lung volumes (in litres; means with standard deviations)

\begin{tabular}{|c|c|c|c|c|}
\hline & $\begin{array}{l}\text { Brace } \\
\text { on }\end{array}$ & $\begin{array}{l}\text { Brace } \\
\text { off }\end{array}$ & $\begin{array}{l}\text { Mean } \\
\text { change } \\
(\%)\end{array}$ & $\begin{array}{l}\text { 95th centile } \\
\text { coefficient of } \\
\text { variation }(\%)\end{array}$ \\
\hline $\begin{array}{l}\text { FVC } \\
\text { FEV } \\
\text { FRC } \\
\text { TLC }\end{array}$ & $\begin{array}{l}2.3(0.6) \\
2.0(0.5) \\
1.7(0.5) \\
3.5(0.7)\end{array}$ & $\begin{array}{l}2.8(0.6) \\
2.4(0.5) \\
2.3(0.5) \\
4.2(0.7)\end{array}$ & $\begin{array}{l}17 \cdot 8^{*} \\
16 \cdot 7^{*} \\
26 \cdot 0^{*} \\
16 \cdot 7^{*}\end{array}$ & $\begin{array}{l}3 \cdot 9 \\
5 \cdot 2 \\
9 \cdot 8 \\
5 \cdot 2\end{array}$ \\
\hline
\end{tabular}

* $\mathrm{p}=<0.001$.

FVC-forced vital capacity; other abbreviations as in table 1.
All overnight oximetry studies, both with an without the brace, showed normal oxygen saturatiog levels of $97-100 \%$ during sleep.

\section{Discussion}

The use of braces in the conservative management of idiopathic scoliosis has been widely practised for the past four decades. Latterly their role has been undef critical review. Recent long term reviews of both Milwaukee and Boston braces concluded that the mean effect of orthotic treatment is to produce a curv $\vec{\omega}$ which is only a few degrees better than the original. . $^{6} 0$

The effect of these restrictive braces on the undere lying lung and chest wall has remained unrecognised? Sevastikoglou et $a l^{10}$ studied the effect of the Milwaukee brace in 26 patients with idiopathic scoliosie and reported that the brace did not alter vital capacit significantly, a conclusion with which we disagree. NPु previous study has examined in detail the change in the subdivisions of total lung capacity associated with bracing.

In our patients there were significant reductions in $\mathrm{FEV}_{1}$ and FVC and in all the subdivisions of TLCO especially FRC (table 2). The reduction in lung volumes appears to be due to a combination of factorso In an attempt to reduce lumbar lordosis the brace ie fitted tightly around the abdomen, thus displacing abdominal contents into the chest, reducing lung volumes, and restricting the downward movement of the diaphragm. With the Boston brace there is also direct pressure effect on the ribcage, which further. restricts respiratory excursion.

It is important to note that there was no difference in the degree of restriction of lung volumes imposed by the two types of brace. Although the Boston brace may vary from centre to centre, depending on indis vidual modification, the Milwaukee brace design if consistent between centres, suggesting that the restrictive effect of these braces is not due to local desig 9 practices.

During inspiration, with descent of the diaphragn the intra-abdominal pressure rises. This pressure if relieved by the outward movement of the abdominat wall and lower rib cage. If these movements ar\& restricted by a brace the intra-abdominal pressure wi $\hat{H}^{\mathrm{N}}$ rise and impose an additional load on the diaphragm? which might have important implications for a chil with diaphragmatic weakness.

Normal subjects have a $20 \%$ reduction in FRC on changing from the sitting to the supine posture." A\$ the mean reduction in FRC for children sitting wit brace on was $26 \%$ we were concerned that there migh be an additional effect while the child slept. This coule be a particular problem during REM sleep, when there 
may be a further fall in FRC owing to relaxation of the intercostal muscles, ${ }^{12}$ and thus a risk of oxygen desaturation. Overnight oxygen saturation was, however, normal in all the six children whose brace restriction was the most severe.

The effects we have found might also have implications for a child who has asthma in addition. During an attack of asthma expiratory flows at all lung volumes are reduced. This is partially offset by an increase in FRC, ${ }^{13}$ achieved by braking expiratory flow with partial glottis closure, increasing the respiratory rate and persisting inspiratory muscle activity during expiration. ${ }^{14,15}$ The restrictive effect of a brace would be likely to limit the child's ability to increase FRC and to breathe at higher lung volumes.

An effect of scoliosis on lung growth has been documented $^{16}$ but the effect of prolonged use of a restricting brace during a period of continuing alveolar growth is unknown.

Although there is general agreement that bracing may be effective in progressive curves of $30-40^{\circ}$, idiopathic scoliosis beginning after the age of 11 years does not appear to carry the risk of cardiorespiratory failure that was hitherto believed. ${ }^{17}$ Thus if braces do not appreciably alter the natural history of adolescent curves, ${ }^{3}$ their use needs to be judiciously reassessed because, as we have shown, they can have a considerable effect on lung volumes, and there may be a potential risk of impairing lung growth or aggravating underlying disorders such as asthma or diaphragmatic weakness.

We gratefully acknowledge the technical assistance of Graeme Johnstone and Andrew Young, Department of Orthotics, and Ian Howlett and Stuart Brown, Department of Thoracic Medicine.

\section{References}

1 Blount WP, Schmidt AC, Keever ED, Leonard ET. The Milwaukee brace in the operative treatment of scoliosis. J Bone Joint Surg [Am] 1958;40-A:511-25.
2 Watts HG, Hall JE, Stanish W. The Boston brace system for the treatment of low thoracic and lumbar scoliosis by the use of a girdle without superstructure. Clin Orthop 1977;126:87-92.

3 Dickson RA. Conservative treatment for idiopathic scoliosis. J Bone Joint Surg [Br] 1985;67-B:176-81.

4 Edgar MA. Editorial. To brace or not to brace. $J$ Bone Joint Surg [Br] 1985;67-B:173-4.

5 SPSSX inc. Users guide. New York: McGraw Hill, 1983.

6 Carr WA, Moe JH, Winter RB, Lonstein JE. Treatment of idiopathic scoliosis in the Milwaukee brace. $J$ Bone Joint Surg [Am] 1980;62-A:599-612.

7 Cochran T, Nachemson A. Long term anatomic and functional changes in patients with adolescent idiopathic scoliosis treated with Milwaukee brace. Spine 1985;10:127-33.

8 Miller JAA, Nachemson AL, Schultz AB. Effectiveness of braces in mild idiopathic scoliosis. Spine 1984;9: 632-5.

9 Mellencamp DD, Blount WP, Anderson AJ. Milwaukee brace treatment of idiopathic scoliosis: late results. Clin Orthop 1977;126:47-57.

10 Sevastikoglou JA, Linderholm H, Lindgren U. Effect of the Milwaukee brace on vital and ventilatory capacity of scoliotic patients. Acta Orthop Scand 1976;47:540-5.

11 Fenn WO, Rahn H, eds. Handbook of Physiology: Respiration. Vol 2. Washington DC: American Physiological Society, 1965:1363-5.

12 Muller NL, Francis PW, Gurwitz D, Levison H, Bryan AC. Mechanism of hemoglobin desaturation during rapid eye movement sleep in normal subjects and in patients with cystic fibrosis. Am Rev Respir Dis 1980;121:463-9.

13 Clark TJH, Godfrey S, eds. Asthma. London: Chapman and Hall, 1983:12-56.

14 Lisboa C, Jardim J, Angus E, Macklem PT. Is extrathoracic airway obstruction important in asthma? $\mathrm{Am}$ Rev Respir Dis 1980;122:115-21.

15 Martin J, Powell E, Shore S, Emrich J, Engel LA. The role of respiratory muscles in the hyperinflation of bronchial asthma. Am Rev Respir Dis 1980;121:441-7.

16 Davies G, Reid L. The effect of scoliosis on growth of alveoli and pulmonary arteries and on right ventricle. Arch Dis Child 1971;46:623-32.

17 Branthwaite MA. Cardiorespiratory consequences of unfused idiopathic scoliosis. $\mathrm{Br} J$ Dis Chest 1986;80:360-9. 